\title{
The effect of Hydroxychloroquine use due to rheumatic disease on the risk of Covid-19 infection and its course
}

\author{
Tanzer Korkmaz ${ }^{1}$, Aslı Şener ${ }^{1}$, Vedat Gerdan ${ }^{2}$, and İlker Kızıloglu ${ }^{3}$ \\ ${ }^{1}$ Affiliation not available \\ ${ }^{2}$ Bakircay Universitesi \\ ${ }^{3}$ Doctor Suat Seren Chest Diseases Training and Research Hospital
}

June 1, 2021

\begin{abstract}
Introduction COVID-19 virus continues to spread and die worldwide. Some studies examining hydroxychloroquine as a prophylactic agent reported that its efficiency and reliability have not been proven so that it must not be used for prophylaxis other than clinical researches. However we think that the results of this study we conducted in a local area will contribute to the literature data. Methods The study was designed as adult patients who were in follow-up taking HC due to their chronic diseases were included. 384 patients who were taking hydroxychloroquine regularly and 299 patients who were not taking hydroxychloroquine were included. The primary outcome of this study is to detect a positive PCR result in patients who received a PCR test and to determine the incidence rate and its course of the disease among those who were taking HC and those who were not taking hydroxychloroquine. Results The majority of the patients (median age: 51.8- range 17-89) were women and housewives. In the patient group using hydroxychloroquine, 22 patients were tested for PCR and only 2 positive patients were found. Covid-19 was detected in $24(8.02 \%)$ of $65(21.73 \%)$ patients who underwent PCR test at Control group (n $=299)$. PCR positivity was statistically lower in the HK group $(\mathrm{n}=2,0.52 \%, \mathrm{n}=24,8.02 \%$, respectively, $\mathrm{p}=0.0001)$. Conclusions Our study supported the fact that there is a lower PCR positivity and symptoms are milder among patients who are using HC regularly, as compared to those who are not using hydroxychloroquine.
\end{abstract}

The effect of Hydroxychloroquine use due to rheumatic disease on the risk of Covid-19 infection and its course

Hydroxychloroquine in Covid-19 and its course

\section{Abstract}

Introduction

COVID-19 virus continues to spread and die worldwide. Some studies examining hydroxychloroquine as a prophylactic agent reported that its efficiency and reliability have not been proven so that it must not be used for prophylaxis other than clinical researches. However we think that the results of this study we conducted in a local area will contribute to the literature data.

\section{Methods}

The study was designed as adult patients who were in follow-up taking HC due to their chronic diseases were included. 384 patients who were taking hydroxychloroquine regularly and 299 patients who were not taking hydroxychloroquine were included.

The primary outcome of this study is to detect a positive PCR result in patients who received a PCR test and to determine the incidence rate and its course of the disease among those who were taking $\mathrm{HC}$ and those 
who were not taking hydroxychloroquine.

Results

The majority of the patients (median age: 51.8- range 17-89) were women and housewives. In the patient group using hydroxychloroquine, 22 patients were tested for PCR and only 2 positive patients were found. Covid-19 was detected in $24(8.02 \%)$ of $65(21.73 \%)$ patients who underwent PCR test at Control group (n $=299)$. PCR positivity was statistically lower in the HK group $(\mathrm{n}=2,0.52 \%, \mathrm{n}=24,8.02 \%$, respectively, $\mathrm{p}=0.0001)$.

Conclusions

Our study supported the fact that there is a lower PCR positivity and symptoms are milder among patients who are using $\mathrm{HC}$ regularly, as compared to those who are not using hydroxychloroquine.

KeyWord: hydroxychloroquine, SARS-CoV-2, Covid-19, prophylaxis, pandemic

\section{İntroduction}

According to Chinese authorities, Severe Acute Respiratory Syndrome-Coronavirus-2 (SARS-CoV-2) (COVID-19), which is a member of the Coronavirus family, has spread rapidly from China to the rest of the world and stopped the life, leaving its mark on the future after 2020. The covid-19 virus that is extracted from patients with pneumonia at Wuhan is an enveloped single-stranded RNA beta-coronavirus. The genomic sequence of Covid-19 shares $79.5 \%$ sequence identity to other coronaviruses that cause Severe Acute Respiratory Syndrome (SARS-CoV). The COVID-19 virus permeates to human alveolar epithelium cells by binding its spike S protein to angiotensin converting enzyme 2 (ACE2) receptor. ${ }^{1}$ The number of cases continues to increase since the COVID-19 virus is rapidly transmitted from human to human. The most important property of this virus whose source has not yet clear is the fact that it can rapidly transmit from human to human through droplets and contact. Thus, it has affected large amounts of people in a short time. This was declared by World Health Organization as a pandemic and emergency. Globally, as of 7 January 2021, there have been 85.509.194 confirmed cases of COVID-19, including 1.868.622 deaths, reported to WHO. ${ }^{2}$ The infection is still uncontrolled in many countries and continues to show its effect. Its course is not yet sufficiently predicted. To reduce the load of Covid-19, effective vaccines and non-pharmacological intervention strategies such as personal, regional, and global isolation or quarantine and full-scale lockdown are adopted. It has a wide clinical spectrum ranging from asymptomatic patients to those with mild symptoms or those requiring hospitalization and even intensive care unit support and may result in death. The majority of Covid-19 patients who are admitted to the intensive care unit have comorbid diseases so they have more hospitalization complications. Therefore, effective treatment can improve the prognosis. ${ }^{3}$ The studies for the treatment of the worldwide spreading Covid-19 virus are in progress. The multi-centered clinical studies in China showed that Chloroquine phosphate, which is a medication primarily used to treat malaria but commonly used for rheumatic diseases, has an apparent efficacy and acceptable reliability for Covid-19-related pneumonia. ${ }^{4}$ Chloroquine is used to treat and prevent Malaria, and an anti-inflammatory agent in the treatment of rheumatoid arthritis, lupus erythematosus, and porphyria cutanea tarda. The studies also revealed that it shows a potential wide-spectrum antiviral activity enhancing the endosomal $\mathrm{pH}$ needed for virus and cell fusion and intervening with glycosylation of the cellular receptors of SARS-CoV. ${ }^{5-7}$ The antiviral and anti-inflammatory activity of hydroxychloroquine (HC) can demonstrate its strong efficiency to treat patients with COVID-19 pneumonia. It was emphasized that there are different opinions about the use of $\mathrm{HC}$ among countries, but this should be supported by further studies. Some studies reported that there is no enough information on this subject and recommended the controlled use of HC, while some studies reported that it should be used in the treatment protocols. ${ }^{4,8}$ Some studies reported that none of the agents are effective to prevent Covid-19. Some studies examining HC as a prophylactic agent reported that its efficiency and reliability have not been proven so that it must not be used for prophylaxis other than clinical researches. However, the results of ongoing studies on prophylaxis (NCT04308668, NCT04303507, NCT04318015, NCT04318444) will enlighten the discussions on this subject. Our study aims to examine the effects of hydroxychloroquine (HC) on the frequency and course of Covid-19 in patients who were ad- 
ministered with $\mathrm{HC}$ for their chronic diseases. ${ }^{9}$ Considering these findings in the current scientific literature, which were mostly contrary to the potential anti-viral effects of these compounds, we think that the results of this study we conducted in a local area will contribute to the literature data.

\section{Method:}

\section{Setting and data source}

The study was designed as an observational, retrospective questionnaire study in which adult patients who were in follow-up in the Department of Rheumatology and taking HC due to their chronic diseases were included. All the patients who were in follow-up in the Department of Rheumatology in our hospital between March and April 2020 were scanned via the "hospital digital registration system". Among them, 384 patients who were taking $\mathrm{HC}$ regularly were included in the study. 299 patients who were not taking HC were included in the control group. The study protocol was reviewed and approved by Izmir Bakircay University Ethics Board. The study protocol conforms to the ethical guidelines of the 1975 Declaration of Helsinki as reflected in a priori approval by the institution's human research committee. Written informed consent was not necessary because no patient data has been included in the manuscript. And the patients are phoned and their verbal consents were obtained.

Study population

We used the data of patients who were in follow-up in the Department of Rheumatology. The patients who were continuing to take $\mathrm{HC}$ in this period were assigned as the experimental group, while those who were not taking $\mathrm{HC}$ were assigned as the control group. The patients who applied to the Rheumatology Department but were not diagnosed with rheumatic disease were excluded. The patients are phoned and their verbal consents were obtained. The consents were obtained verbally since it was not appropriate for the patients to come to the hospital in terms of contamination risk in the Covid-19 pandemic process. It was emphasized that their data will not be shared in any way. The information on their demographical characteristics (age, gender, occupation), primary diseases, other medications than HC they used, the dose of HC they received, whether they had Covid-19, whether they were administered with PCR test as a potential case, if so the results of the test (From the individual's self-declaration or central result system referred to as "PHMS" Public Health Management System), whether people who they were living together or those with whom they had close contact were diagnosed with Covid-19 and received Covid-19 treatment, were recorded. Information on the course of disease (follow-up at home, hospitalization, intensive care unit, exitus) was collected from the hospital registration system or the patients themselves.

Main outcomes and measures

The primary outcome is to detect a positive PCR result in patients who received a PCR test and to determine the incidence rate of the disease among those who were taking $\mathrm{HC}$ and those who were not taking HC.

Statistical analysis

The statistical analysis was performed using SPSS 22 (SPSS Inc., Chicago, IL) software program. The demographic data were analyzed based on the means and standard deviation and the Chi-square test was used for group comparisons. Continuous data were expressed as mean \pm standard deviation and categorical parameters were computed as percentages, where appropriate. If $\mathrm{p}<0.05$, then the result was considered statistically significant.

\section{Results}

The patients who were taking $\mathrm{HC}$ agents due to rheumatic disease and those in the control group were examined (Figure 1). It was seen that the majority of patients were female and housewives (Figure 2, Table 1). The median age was 51.8 years (from 17 to 89 ) and the majority of them were in the 50-60 age band (Figure 3). It was found that $56(14.6 \%)$ patients had comorbidity $(14.6 \%)$, while $34(8.9 \%)$ had diabetes and $9(2.3 \%)$ had hypertension $(2.3 \%)$. All the patients were taking HC $1 \mathrm{x} 1 /$ day or $2 \mathrm{x} 1$ /day. Thirty-two $(8.33 \%)$ patients who were included in the study could not be reached by phone. Based on the phone calls, 
the number of patients who were in close contact with Covid-19 patients was 15 (3.9\%). The number of the patients who underwent Covid-19 examination for any reason was found to be $5.72 \%(22 / 384)$. Only $2(0.52 \%)$ patients under our observation were diagnosed with Covid-19. Both of them were mild cases and without hospitalization. When we examined 299 patients who were under follow-up because of their rheumatic diseases but were not taking HC, PCR positivity was detected in $24(8.02 \%)$ of $65(21.73 \%)$ patients who received a PCR test. It was also found that two patients who were not using $\mathrm{HC}$ died due to Covid-19. When we compared the PCR positivity rate between the patients who were taking $\mathrm{HC}(\mathrm{n}=2)$ and those who were not using $\mathrm{HC}(\mathrm{n}=24)$, we found that the PCR positivity was statistically lower in the HC group (Respectively $\mathrm{n}=2$ 0.52\%, $\mathrm{n}=248.02 \%, \mathrm{p}=0.0001$ ).

One of the Covid-19 patients was a 42-year-old teacher and the other was a 48-year-old housewife. Both of them had connective tissue disease. The phone calls with the patients revealed that the teacher patient has not worked during the pandemic period but her husband contacted other patients (for about 1 hour) when their daughter got sick. Then first her husband and then she was diagnosed with Covid-19 (PCR and thorax CT positive). She healed at home with mild myalgia and fever which lasted for few days. The other patient who was a housewife had no contact with a confirmed Covid-19 patient. But her husband was a plumber and had a plumbery. She had an irritable cough and her thorax CT was compatible with viral pneumonia but healed at home without any complication.

\section{Discussion}

Severe acute respiratory syndrome (SARS) is caused by a newly discovered coronavirus (Covid-19). No effective prophylactic or post-exposure therapy is currently available. ${ }^{10}$ Some disease-modifying agents commonly used to treat patients with rheumatic diseases/autoimmune disorders, such as HC and colchicine, are explored as potential treatments for COVID-19. However, their prophylactic roles are still not certain. ${ }^{11}$ Hydroxychloroquine was first approved for medical use in the USA in $1955 .{ }^{1}$ The studies showed that these molecules inhibit viral DNA and RNA polymerase enzymes and also inhibit viral protein glycosylation and viral proliferation. The virus binds to target cells by angiotensin-converting enzyme 2 (ACE2). HC was preferred in SARS treatment because it can inhibit the terminal glycosylation of ACE2 and modify cytokine release. It was thought that it can be used in Covid-19 treatment. ${ }^{6}$ It was observed that in clinical use, HC prevented the progress of virus-related pneumonia. In March 2020 hydroxychloroquine (HC) was reported to have a positive effect in a Covid-19 treatment open-label non-randomized clinical trial. In this study, hydroxychloroquine and azithromycin were used to prevent bacterial infection, and no treatment was applied to the negative control group. 20 of 36 patients were administered with azithromycin and $600 \mathrm{mg} / \mathrm{day} \mathrm{HC}$. A significant decrease in viral load was found on day 6 , as compared with the control group. ${ }^{12}$ However, Rosendaal reported that there were great methodological deficits in this study and the results did not support the results on the efficiency of hydroxychloroquine in Covid-19. ${ }^{13}$ Similarly, Goldman et al. stated in their comment that randomized controlled studies are needed for the data to be significant. ${ }^{14}$ The difference of our study from the study by Gautret et all. is that our study was not an intervention and treatment study and it was only an observational study for patients with HC use history. Currently, there is still no suggestion to use antiviral agents as prophylaxis for Covid-19 but many studies are in progress to evaluate the effects of treatment as well as prophylaxis. ${ }^{9}$ We hope that the results of the ongoing studies will give us more insight into prophylaxis and help with prophylaxis agents, which will reduce the rapid spread of the disease. Another study reviewed all available resources to overview the potential role of $\mathrm{HC}$ in preventing the spread of COVID-19 and to determine a reasonable schedule for its use as prophylaxis. It was stated that there were very limited clinical data on the prophylactic role of HC. It was reported that HC is used for prophylaxis because it showed reductions of exacerbation of pneumonia, duration of symptoms, and delay of viral clearance, all in the absence of severe side effects. As a result, they said that further information can be obtained only by wide prospective randomized studies. ${ }^{15}$ We hope that our data and a meta-analysis of the results of similar observational studies and ongoing randomized studies serve to justify the large-scale use of prophylactic HC. Although research on the use of HC in the control of COVID-19 is still preliminary, the potential use of these drugs is supported by factors such as their usability in the treatment of rheumatic and infectious diseases such as malaria, low cost and history of use. Furthermore, HC is increasingly pre- 
ferred because it is a less toxic derivative of CQ and showed better results in inhibiting the proliferation of SARS-CoV-2. ${ }^{16}$ The review study by Preira reported that a meta-analysis was conducted to investigate the effects of using CQ and $\mathrm{HC}$ in 16,679 patients with rheumatic disease, but their effects on cardiomyopathy and neuropathy were not confirmed. In the systematic literature review, he examined cardiac complications associated with $\mathrm{CQ}$ and $\mathrm{HC}$ and noted that cardiac disorders related to $\mathrm{CQ}$ or $\mathrm{HC}$ were rare, but a prolonged administration resulted in serious adverse effects. He emphasized that there is no evidence on the preventive effect of $\mathrm{HC}$ on Covid-19 and suggested that sharing $\mathrm{HC}$ use information will lead to healthier results. ${ }^{16}$ Our main purpose was to determine whether $\mathrm{HC}$ can be used or not, instead of reaching a conclusion. We aim to share our data that will likely to contribute studies with larger participation.

In a data analysis conducted in Israel, all COVID-19 cases were screened and it was found that 36 patients were using $\mathrm{HC}$ regularly and only three (0.23\%) patients were COVID-19 positive. ${ }^{11}$ In our study, 384 patients who were using $\mathrm{HC}$ were screened and it was found that $2(0.52 \%)$ of 22 patients who were examined were Covid-19 positive, and 24 (8.02\%) of 65 patients who were not using $\mathrm{HC}$ and examined were Covid-19 positive. In the study by Boulware et al., randomly selected 821 asymptomatic Covid- 19 patients were administered with placebo and $\mathrm{HC}$ after the exposure. They reported that there was no difference between the two groups and no serious adverse effect was seen. ${ }^{17}$ Rajasingham et al. did not find a significant decrease in the incidence of Covid-19 based on the prophylaxis results of 1483 health professionals before the HC exposure (400 mg $\mathrm{HC}$ twice a week and $400 \mathrm{mg} \mathrm{HC}$ and placebo twice a week). ${ }^{18}$ Abella et al. conducted a randomized, double-blind, placebo-controlled study on a population of health professionals but early stopped the study since they predicted that there would be no significant difference. However, they did not report any serious side effects. ${ }^{19}$ Although there are no many studies suggesting the use of $\mathrm{HC}$ for prophylaxis, more reliable data will be obtained if all researchers shared their data and the results of randomized controlled studies are provided. It seems that; Covid-19 will continue to be a threat to the world.

\section{Conclusion}

Our study supported the fact that there is a lower PCR positivity and symptoms are milder among patients who are using HC regularly, as compared to those who are not using HC. Whether prior exposure prophylaxis is effective in populations at high risk is a separate question and studies are continuing and will continue. If our results are reproduced in other ongoing studies, our results will be verified.

\section{Limitations}

We do not know the rate of patients who are using HC regularly among all suspected Covid-19 cases. We examined only the HC patients in an isolated area. The lack of precisely recorded comorbidities in our all data directed us to only the patients who were registered and using $\mathrm{HC}$ regularly. Another limitation was the lack of data of patients who could not be reached by phone and of those in the control group. Another limitation was the fact that the contact histories of patients were evaluated only based on the self-declaration. It is difficult to determine who contacted Covid-19 patients, which doesn't make much sense today.

Author contributions: All the authors contributed intellectually and directly to the preparation of this article, and read and approved the current version of the article for publication.

Conflicting interests: The Author(s) declare(s) that there is no conflict of interest.

\section{References}

1. Yang Y, Islam MS, Wang J, Li Y, Chen X. Traditional Chinese Medicine in the Treatment of Patients Infected with 2019-New Coronavirus (SARS-CoV-2): A Review and Perspective. International journal of biological sciences 2020;16(10):1708-1717.

2. Dashboard WCDC-: https://covid19.who.int. Data last updated: 2021/1/7, 10:18am CET 2021.

3. Huang C, Wang Y, Li X, Ren L, Zhao J, Hu Y, et al. Clinical features of patients infected with 2019 novel coronavirus in Wuhan, China. Lancet 2020; 395(10223):497-506. 
4. Gao J TZ, Yang X. Breakthrough: Chloroquine phosphate has shown apparent efficacy in treatment of COVID-19 associated pneumonia in clinical studies. BioScience Trends /Advance Publication 2020;14(1): 72-73. doi.org/10.5582/bst.2020.01047.

5. Savarino A, Boelaert JR, Cassone A, Majori G, Cauda R. Effects of chloroquine on viral infections: an old drug against today's diseases? Lancet Infect Dis 2003; 3(11):722-727.

6. Wang M, Cao R, Zhang L, Yang X, Liu J, Xu M, et al. Remdesivir and chloroquine effectively inhibit the recently emerged novel coronavirus (2019-nCoV) in vitro. Cell Res 2020;30(3):269-271.

7. Yao X, Ye F, Zhang M, Cui C, Huang B, Niu P, et al. In Vitro Antiviral Activity and Projection of Optimized Dosing Design of Hydroxychloroquine for the Treatment of Severe Acute Respiratory Syndrome Coronavirus 2 (SARS-CoV-2). Clin Infect Dis 2020;71(15):732-739. doi: 10.1093/cid/ciaa237.

8. Infectious Diseases Society of America Guidelines on the Treatment and Management of Patients with COVID-19. https://www.idsociety.org/practice-guideline/covid-19-guideline-treatment-andmanagement/ (Accessed on April 13, 2020).

9. Agrawal S GAD, Gupta N. Emerging prophylaxis strategies against COVID-19. Monaldi Archives for Chest Disease 2020; 90:1289.

10. Vincent MJ, Bergeron E, Benjannet S, Erickson BR, Rollin PE, Ksiazek TG, et al. Chloroquine is a potent inhibitor of SARS coronavirus infection and spread. Virology Journal 2005; 2 (1):69.

11. Gendelman O, Amital H, Bragazzi NL, Watad A, Chodick G. Continuous hydroxychloroquine or colchicine therapy does not prevent infection with SARS-CoV-2: Insights from a large healthcare database analysis. Autoimmun Rev 2020; 19(7):102566-102566.

12. Gautret P LJ, Parola P, Hoang T, Meddeb L, Mailhe M, Doudier B, et al. Hydroxychloroquine and azithromycin as a treatment of COVID-19: results of an open- label non-randomized clinical trial. Int J Antimicrob Agents 2020;56(1):105949. doi: 10.1016/j.ijantimicag.2020.105949.

13. Rosendaal FR. Review of: "Hydroxychloroquine and azithromycin as a treatment of COVID-19: results of an open-label non-randomized clinical trial Gautret et al. Int J Antimicrob Agents 2020;56(1):106063. doi:10.1016/j.ijantimicag.2020.105949.

14. Goldman JD, Diaz G, Urba WJ. Use of hydroxychloroquine in combination with azithromycin for patients with COVID-19 is not supported by recent literature. Int J Antimicrob Agents 2021; 57(1):106174.

15. Monti M, Vertogen B, Masini C, Donati C, Lilli C, Zingaretti C, et al. Hydroxychloroquine as Prophylaxis for COVID-19: A Review. Frontiers in pharmacology 2020;11:605185-605185.

16. Pereira BB. Challenges and cares to promote rational use of chloroquine and hydroxychloroquine in the management of coronavirus disease 2019 (COVID-19) pandemic: a timely review. J Toxicol Environ Health B Crit Rev 2020;23(4):177-181.

17. Boulware DR, Pullen MF, Bangdiwala AS, Pastick KA, Lofgren SM, Okafor EC, et al. A Randomized Trial of Hydroxychloroquine as Postexposure Prophylaxis for Covid-19. New England Journal of Medicine 2020;383(6):517-525.

18. Rajasingham R, Bangdiwala AS, Nicol MR, Skipper CP, Pastick KA, Axelrod ML, Pullen MF, Nascene AA, Williams DA, Engen NW et al: Hydroxychloroquine as Pre-exposure Prophylaxis for Coronavirus Disease 2019 (COVID-19) in Healthcare Workers: A Randomized Trial. Clinical Infectious Diseases 2020; ciaa1571. doi.org/10.1093/cid/ciaa1571.

19. Abella BS, Jolkovsky EL, Biney BT, Uspal JE, Hyman MC, Frank I, Hensley SE, Gill S, Vogl DT, Maillard I et al: Efficacy and Safety of Hydroxychloroquine vs Placebo for Pre-exposure SARS-CoV-2 Prophylaxis Among Health Care Workers: A Randomized Clinical Trial. JAMA Internal Medicine 2021;181(2):195-202. doi:10.1001/jamainternmed.2020.6319 
Figure 1. Working flow chart

Figure 2. Gender distribution of patients using hydroxychloroquine

Figure 3. Distribution of patients using hydroxychloroquine by age groups

Table1. Occupational distribution of patients

\begin{tabular}{lll}
\hline Occupation & $\mathbf{n}$ & $\mathbf{\%}$ \\
Housewife & 233 & 60.7 \\
Retired & 47 & 12.2 \\
Officer* & 24 & 6.3 \\
Self-employment & 34 & 8.9 \\
Student & 3 & 0.8 \\
Worker & 30 & 7.8 \\
Missing & 13 & 3.4 \\
Total & 384 & 100.0 \\
\hline
\end{tabular}

* Civil servants include 9 healthcare workers (4 nurses, one doctor, 4 others) and 5 teachers)

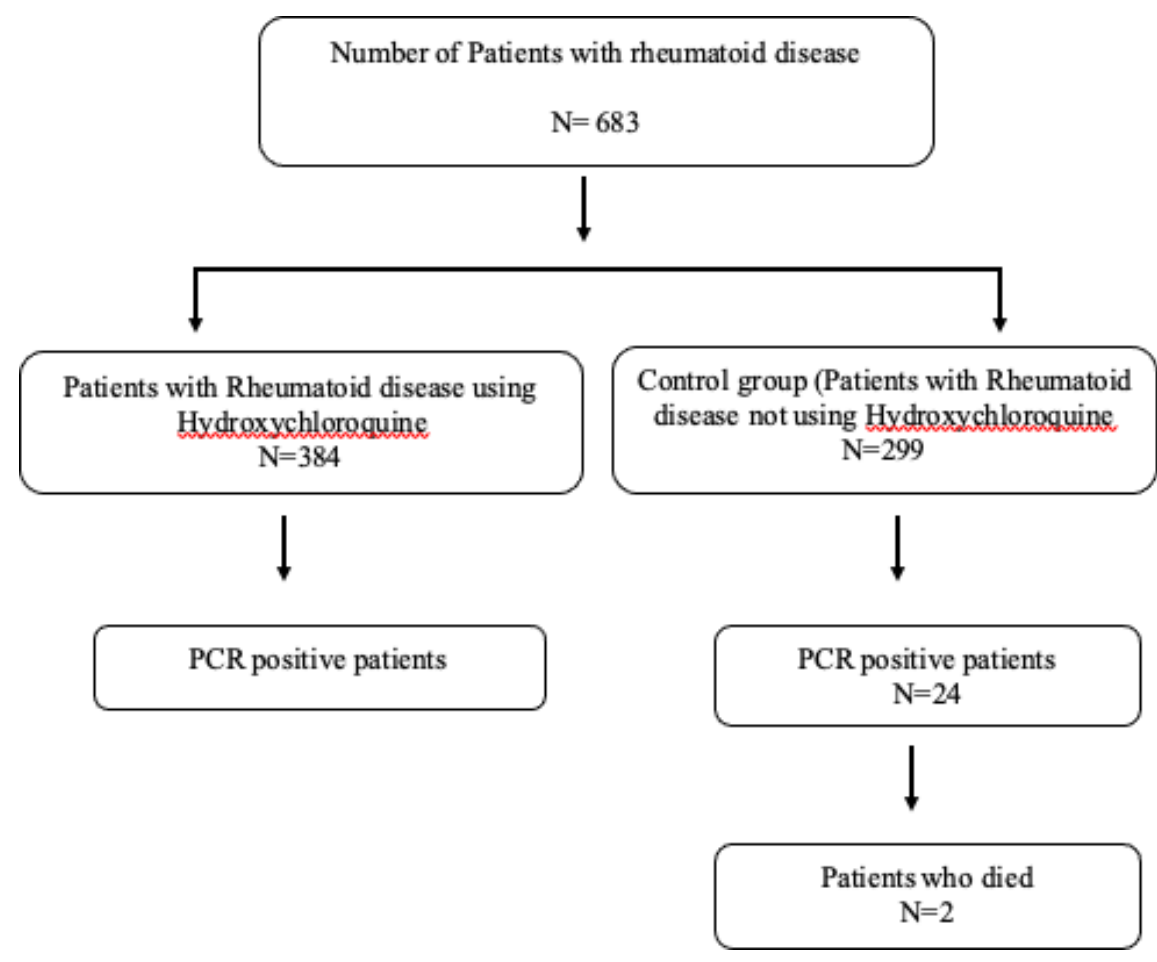



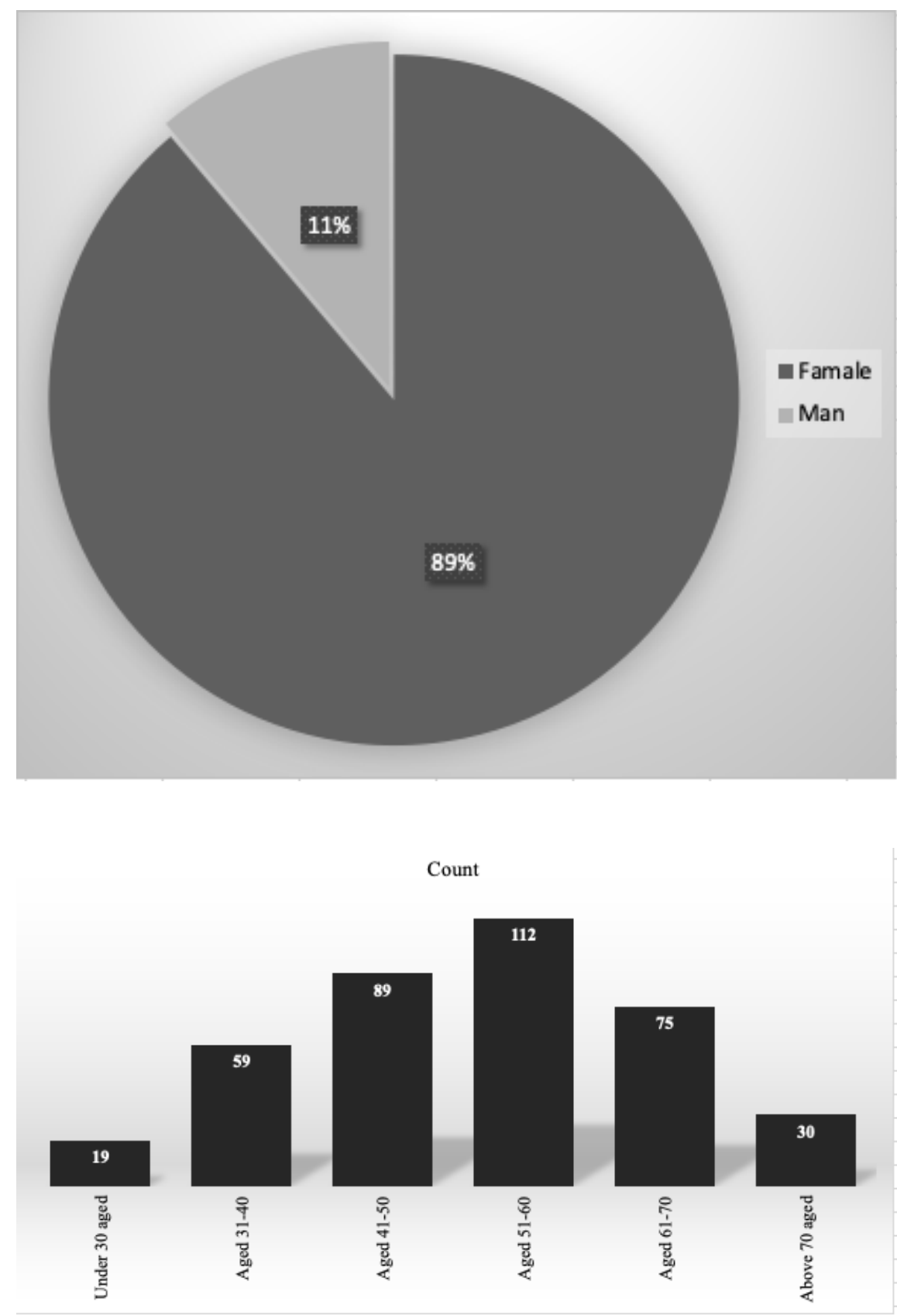

\section{Hosted file}

Table1.docx available at https://authorea.com/users/417382/articles/524514-the-effect-ofhydroxychloroquine-use-due-to-rheumatic-disease-on-the-risk-of-covid-19-infection-andits-course 